\title{
EDITORIAL
}

\section{Promoting Scientific Research for Economic Development}

Scientific research generally covers basic research and applied research, although the demarcation between these two is not well defined. In general, basic research develops or generates basic scientific knowledge explaining the characteristics properties of nature without violating the known fundamental principles. In contrast, the original work of the applied researchers tests the predictions of basic science research or develops novel methods or ways to transform the basic scientific knowledge into specific pre-determined applications to solve practical problems.

The key benefit for developing countries to pursue applied and basic research is the potential impact on economic development. Governments in many countries, including developing countries, directly support both basic research and applied research. If the government plans to foster and harness research and development (R\&D) for economic development, there are a number of potential tools that are being utilized, including direct funding for research programmes at government research institutes and universities. Funding with tax incentives may also be available to private-sector researchers who engaged in specific collaborative $R \& D$ projects.

The establishment of appropriate policies by governments to support research could be a vital driver of the economic development of their countries, as highlighted below. Whatever the method used to prioritize and channel the government support for innovation and $R \& D$ it will be more effective if the support is recognized it as a long-term investment. In general, the time lag from basic research to commercial applications to gain the ultimate economic benefits can be very long. As an example, today's widespread commercialization of biotechnology including the development of Covid-19 vaccines within a relatively short span of time was based, in part, on key research findings made in the 1950s.

Government support for R\&D in any country presumes sufficient national capacity of trained human resources to engage in productive research on themes of national importance. That capacity on human resources depends on the availability of qualified scientists, engineers and other trained technical support staff, which in turn depends on the country's education policy, especially in science-related disciplines.

Promoting R\&D and innovation activity is expected to benefit national economic development through the creation of new knowledge and improved technology while at the same time training a highly skilled S\&T workforce. Cross-national analyses have shown that the size of the scientific workforce and the R\&D capacity of a nation have a direct relationship with the country's economic development.
One anticipated and essential policy response, especially from governments of developing countries, would be to substantially strengthen the intellectual property rights of research scientists, who generate new and innovative ideas, strong and long-lasting claims to the economic benefits of their discoveries.

Research and developments (R\&D) are not only for well-established industries. Small and medium-sized enterprises (SMEs) can leverage R\&D to improve their products and services rapidly to compete in the global market and develop an innovative idea of a new product or a service to reality. SMEs are indeed recognized as the engines of global economic growth, especially in developing countries. Their continued growth is becoming a significant component in the development of the economy and increases the employment opportunities of any country. Under the pressure of globalized competitive market forces, producers are expected to continuously innovate and upgrade their production lines enhancing the efficiency and the quality of their existing products. Towards that end, research and development (R\&D) in the SME sector could be a viable option to sustain and make the operations of SMEs much more efficient and competitive in the global market. Through such R\&D, they will not hesitate to upgrade and improve their age-old methods to produce traditional products firmly rooted in the country with unique properties. In this way, these producers/exporters will soon recognize the commercial potential of $R \& D$ on further developing their "traditional products of national/ regional origin".

As an example, in the case of the agro-industrial sector, where they have to compete with other countries for exporting their agricultural commodities as producers and small and medium-sized companies, the value addition through R\&D and innovation signifies a major opportunity to promote their generic and standardized products in the competitive export market. Small and medium agroindustries in developing countries face several challenges such as poor transport infrastructure, lack of packing materials and lack of technical expertise. These challenges reduce profitability and competitiveness. Government support and relevant R\&D should address such issues.

It should be noted the development through applied research alone is limited without further advancement of basic research. To sustain the R\&D system of any country, the basic research platform must be developed in parallel with the applied research. Therefore, to face competitive challenges in the world of high technology, any country, developed or developing, needs to support scientists conducting both, basic research as well as applied science research. It is well known that the basic scientific research in 
Thermodynamics, Electricity and Magnetism, Condensed Matter Physics, Semiconductor Physics, Chemistry, Biology and Bio-technology, Mathematics, Theoretical subjects and other fundamental subjects had made a significant contribution to the discovery of new knowledge in the history of science opening up many pathways for the rapid development of applied research leading to technological, industrial and economic development in the modern world.

With the invention of computers and the internet, the rapid development of communication technology during approximately 1970-2010, an unprecedented industrial revolution happened in the world. At present, research is underway using one of the most fundamental knowledge in basic sciences, namely in Quantum Mechanics, attempting to develop much faster and more efficient quantum computers. Probing the quantum mechanical properties of matter, a new paradigm of computing called quantum computing, with new programming languages, will probably make a significant development creating more opportunities for young scientists to work around the world and collaborate internationally. Several industries are already racing to build a commercially viable quantum computer, perhaps 100 million times faster than the fastest classical computer available today. It is another fascinating attempt of adopting basic science knowledge, invented about a hundred years ago, to applied research today, leading to new technology, perhaps creating the pathway for the next industrial revolution. All countries, developed and developing, must prepare and train scientists and students to face the challenges of new technology in the future for their economic development.

Research needs to be considered as an investment, not as an expense. A large amount of funds is spent annually all over the world on R\&D and innovation to upgrade the products and services in a highly competitive global economy. It is equally important for all countries, developed and developing, to make sure that the younger generation is adequately equipped with basic scientific knowledge and research training to face future challenges. Without this preparation, especially the developing countries will lag behind by a couple of decades as already happened in the past.

W.G.D. Dharmaratna University of Ruhuna, Sri Lanka Email:dharma@phy.ruh.ac.lk 